\title{
MicroRNA-423-5p targets O-GIcNAc transferase to induce apoptosis in cardiomyocytes
}

\author{
PENG LUO, TAO HE, RONGJIAN JIANG and GANG LI \\ Department of Cardiology, Sichuan Academy of Medical Sciences and Sichuan Provincial People's Hospital, \\ Chengdu, Sichuan 610072, P.R. China
}

Received April 17, 2014; Accepted February 11, 2015

DOI: $10.3892 / \mathrm{mmr} .2015 .3491$

\begin{abstract}
Congestive heart failure (CHF) remains the single most prevalent cause of mortality and morbidity; therefore, the identification of novel biomarkers is required for the diagnosis of CHF. The aim of the present study was to examine whether microRNA (miR)-423-5p is a novel biomarker for CHF, which was defined by the circulatory biomarker pro-brain natriuretic peptide (BNP). Samples of plasma from patients with $\mathrm{CHF}$ and healthy controls were collected and used for quantitative polymerase chain reaction analysis. In addition, the O-GlcNAc transferase (OGT) 3' untranslated region was cloned and used for a luciferase assay. The effects of the expression of miR-423-5p in cardiomyocytes were determined using western blotting and terminal deoxynucleotidyl transferase-deoxyuridine triphosphate nick-end labeling analyses. The results demonstrated that miR-423-5p was associated with $\mathrm{CHF}$ and the expression levels of proBNP; in addition, OGT was found to be a direct target of miR-423-5p. The expression of miR-423-5p significantly regulated the expression of OGT and its associated downstream targets and induced apoptosis in the cardiomyocytes. Therefore, the results of the present study indicated that miR-423-5p was involved in CHF via the direct targeting of OGT and the induction of apoptosis in cardiomyocytes.
\end{abstract}

\section{Introduction}

The post-translational $\beta$-O-linkage of $\mathrm{N}$-acetylglucosamine (O-GlcNAc) to cellular proteins represents one signaling pathway, which has been implicated in the pathophysiology of cardiovascular disease (1). O-GlcNAc transferase (OGT), which adds O-GlcNAc to proteins, has been demonstrated to be required for cell division and embryogenesis (2). Cardiomyocytes ablation of OGT induces heart failure and OGT is required

Correspondence to: Dr Gang Li, Department of Cardiology, Sichuan Academy of Medical Sciences and Sichuan Provincial People's Hospital, 32 West $2^{\text {nd }}$ Section, $1^{\text {st }}$ Ring Road, Chengdu, Sichuan 610072, P.R. China

E-mail: 250325743@qq.com

Key words: congestive heart failure, microRNA-423-5p, O-GlcNAc transferase, cardiomyocytes as part of the endogenous compensatory response to infarctinduced heart failure (3). In developed countries, the single most prevalent cause of mortality and morbidity is congestive heart failure (CHF) (4). CHF accounts for $\sim 5,000,000$ hospitalizations annually in developed nations, which results in the treatment of $\mathrm{CHF}$ being a major expenditure in the health care budgets of all developed nations, and is also becoming an increasing expense for those of numerous developed nations. CHF is known to occur as a common result of a wide range of etiologies; however, the propensity to develop $\mathrm{CHF}$ varies significantly following a given insult $(4,5)$. This is considered to be due to responses, including inherited variation in myocardial and vascular responses. However, the factors underlying the ultimate development of $\mathrm{CHF}$ remain to be fully elucidated. The clinical management of $\mathrm{CHF}$ is enabled by the identification of circulating biomarkers, including brain natriuretic peptide (BNP) $(6,7)$. However, there remains a requirement for the identification of novel circulating biomarkers as objective indicators of $\mathrm{CHF}$, which can be readily and reliably measured.

MicroRNAs (miRNAs) were first identified in mammals as a large class of evolutionarily conserved small, non-coding RNAs. These are involved in the regulation of gene expression at the post-transcriptional level through targeted interactions with the 3'-untranslated region (UTR) of messenger RNA (mRNA) transcripts (8). miRNAs are important regulators of a wide range of biological processes by affecting protein translation (8). In addition, miRNAs can be efficiently suppressed for prolonged periods using antisense technologies, which has been of increasing interest due to its potential therapeutic use in cardiovascular diseases through the targeted inhibition of specific miRNAs (9). A study in 2008 revealed that miRNAs were also present in blood, where they were detected in plasma, platelets, erythrocytes and nucleated blood cells. Plasma miRNAs were found to remain stable, even under conditions of stress, including boiling, low or high $\mathrm{pH}$, long-term storage at room temperature and multiple freeze-thaw cycles (10-12). These characteristics suggest that miRNAs may be a suitable biomarker for disease diagnosis.

In previous years, miR-423-5p has been investigated widely as a potential biomarker for heart diseases. Array investigations have reported that miR-423-5p is upregulated in human failing myocardium (13). Tijsen et al (14) demonstrated that circulating levels of miR-423-5p are increased in patients with clinical heart failure, defined by the Framingham criteria and elevated 
pro-brain (pro)BNP levels. This indicated that the levels of miR-423-5p levels were associated with proBNP and ejection fraction in this patient group (14). However, the association between miR-423-5p and CHF and the underlying mechanism remain to be elucidated. Therefore, elucidating the direct target of miR-423-5p and the associated pathways in cardiomyocytes is essential for understanding the association between miR-423-5p and heart disease, and to determine why miR-423-5p fails as a biomarker for systemic ventricular and left ventricular remodeling $(15,16)$.

The present study aimed to examine the role of miR-423-5p in the development of CHF through investigating the expression of miR-423-5p in the plasma of patients with CHF and healthy volunteers. The expression levels of miR-423-5p, proBNP, OGT and the downstream targets of OGT, and apoptosis in the cardiomyocytes were examined.

\section{Materials and methods}

Clinical study. The present study was approved by the ethics committee of Sichuan Academy of Medical Sciences \& Sichuan Provinicial People's Hospital (Chengdu, China). Human plasma samples were obtained with informed consent under a general waiver by the Academic Medical Center institutional review board for the proper secondary use of human material. Plasma samples were obtained from the patients and healthy volunteers at the People's Hospital of Sichuan Province and experiments were performed on samples at the Sichuan Academy of Medical Science. Written informed consent was obtained from the patients and volunteers.

Definition of CHF diagnosis. The subjects were classified as CHF cases in accordance with the Framingham criteria for CHF diagnosis (17) and if they had circulating proBNP levels of $>1,000 \mathrm{pg} / \mathrm{ml}$. The subjects were classified as non-CHF cases if the clinical diagnosis excluded CHF and if circulating proBNP levels were below the age-associated cut-off points. In total, 51 of the 74 subjects screened for CHF fulfilled the criteria.

Cell culture and transfection. Mouse cardiomyocytes were obtained from Shanghai Cell Bank (Shanghai, China). The cardiomyocytes were cultured in Dulbecco's Modified Eagle's Medium (DMEM) containing 10\% fetal bovine serum (FBS; Gibco-BRL, Carlsbad, CA, USA). The cells were maintained in a humidified atmosphere containing $5 \% \mathrm{CO}_{2}$ at $37^{\circ} \mathrm{C}$. Cell transfection was performed using FuGENE ${ }^{\circledR}$ HD Transfection Reagent (Roche Diagnostics, Indianapolis, IN, USA), according to the manufacturer's instructions. Briefly, the cardiomyocytes were seeded into six-well plates at a density of $2 \times 10^{5}$ cells/well and cultured for $24 \mathrm{~h}$ to reach $70-80 \%$ confluence. Plasmids $(2 \mu \mathrm{g})$, encoding mouse (m)-miR-423-5p and miR-Ctrl, were diluted in $100 \mu \mathrm{l}$ media without serum. Subsequently, $5 \mu \mathrm{l} \mathrm{FuGENE}{ }^{\circledR}$ HD Transfection Reagent was added to tubes containing the diluted DNA, mixed, incubated with the transfection complex for $15 \mathrm{~min}$ at room temperature and then added to the six-well plates. A total of 24 or $48 \mathrm{~h}$ post-transfection the cells were collected for total mRNA and protein extraction. miR-Ctrl was transfected for $48 \mathrm{~h}$. Medium (DMEM containing 10\% FBS) alone was used as the blank agent. Cardiomyoctes was placed in a six-well plate at a density of $2 \times 10^{5}$ cells/well. After $24 \mathrm{~h}$, puromycin (Beyotime
Biotechnology, Shanghai, China) was used to treat cardiomyoctes at $0,5,10,20$ or $40 \mu \mathrm{M}$. The cell vitality was observed in the following 48-72 $\mathrm{h}$. The terminal concentration was found to be $20 \mu \mathrm{M}$. Consequently, after plasmid transfection for $48 \mathrm{~h}, 20 \mu \mathrm{M}$ puromycin was added to treat cardiomyoctes. The surviving cells were selected as the stable cells which expressed the plasmid.

Bioinformatics analysis. To analyze the potential targets of miR-423-5p, online miRNA databases were used. TargetScan (http://www.targetscan.org/) and microRNA.org (http://www. microrna.org/microrna/getGeneForm.do) were used to predict the potential targets of miR-423-5p, and O-GlcNAc was identified as a target. Further study was performed to determine whether O-GlcNAc is the direct target of miR-423-5p.

Luciferase assays. The miR-423-5p binding site was synthesized and cloned into an Ambion pMIR-REPORT vector (Ambion Life Technologies, Carlsbad, CA, USA) to generate pMiRluc-423-5p. Briefly, each miRNA and plasmid oligonucleotide was annealed at $90^{\circ} \mathrm{C}$ for $3 \mathrm{~min}$, cooled to $37^{\circ} \mathrm{C}$, and incubated for $1 \mathrm{~h}$. The annealed dsDNA oligonucleotides were ligated between HindIII and SacI (Fermentas, Thermo Scientific, Waltham, MA, USA) sites on the pMIR-REPORT vector. The resulting recombinant plasmids were named pMIR-miR-423-5p and pMIR-miR-Ctrl. The two constructs were verified by DNA sequencing (Invitrogen Life Technologies, Carlsbad, CA, USA). Plasmids were extracted using EndoFree Plasmid Giga kits (Qiagen GmbH, Hilden, Germany) from DH5 $\alpha$ (Genewiz, Suzhou, China) Escherichia coli transformants and stored at $-20^{\circ} \mathrm{C}$ until further use. The concentration was determined by measuring the A260/A280 ratio using a Thermo ND 2000 spectrophotometer (Thermo Scientific). The 3'-UTRs of O-GlcNAc transferase (OGT) containing miR-423-5p binding sites were amplified and cloned into the same vector to generate pMiRluc-OGT. The reporter was co-transfected with a cytomegalovirus $\beta$-galactosidase vector using FuGENE HD ${ }^{\circledast}$ (Roche Diagnostics). Luciferase activity was measured $4 \mathrm{~h}$ later using a Luciferase Reporter assay (Promega Corporation, Madison, WI, USA). Values were normalized to the activity of $\beta$-galactosidase.

26Sproteasomeactivityassay. The function of the $26 \mathrm{~S}$ proteasome was assayed, as described previously (17). Briefly, the cells were seeded at a density of $2 \times 10^{5}$ cells/well and cultured for $24 \mathrm{~h}$, until they had reached $70-80 \%$ confluence. The cells were then washed with phosphate-buffered saline (ZsBio, Beijing, China), and then with buffer I, containing I50 mM Tris (pH 7.4), 2 mM dithiothreitol, $5 \mathrm{mM} \mathrm{MgCl}_{2}$ and $2 \mathrm{mM}$ adenosine triphosphate (ATP)], and pelleted by centrifugation (Mini Centrifuge \#166-0623; Bio-Rad Laboratories, Inc., Hercules, CA, USA) at $200 \mathrm{x}$ g for 5 min. Glass beads (Beyotime Biotechnology) and homogenization buffer, containing $50 \mathrm{mM}$ Tris ( $\mathrm{pH}$ 7.4), $1 \mathrm{mM}$ dithiothreitol, $5 \mathrm{mM} \mathrm{MgCl}_{2}, 2 \mathrm{mM}$ ATP and $250 \mathrm{mM}$ sucrose, were added and vortexed (MixPlus; Aibenseng Bio, Hefei, China) for $1 \mathrm{~min}$. The beads and cell debris were removed by centrifugation at $1,000 \mathrm{x} \mathrm{g}$ for $5 \mathrm{~min}$ and then at $10,000 \mathrm{x} \mathrm{g}$ for $20 \mathrm{~min}$. The protein concentrations were determined using a bicinchoninic acid assay (Pierce Biotechnology, Inc., Rockford, IL, USA), according to the manufacturer's instructions. The protein from each sample $(100 \mu \mathrm{g})$ was diluted with buffer I to a final volume of 1,000 $\mu 1$, and Suc-LLVY-7-amido-4-methylcoumarin fluoro- 
genic proteasome substrate (chymotrypsin-like; Sigma-Aldrich, St., Louis, MO, USA) was added to a final concentration of 80 $\mu \mathrm{M}$ in $1 \%$ dimethyl sulfoxide (Sigma-Aldrich). In order to access the $20 \mathrm{~S}$ function, the buffer I was replaced with an ATP-free buffer containing $20 \mathrm{mM}$ HEPES (pH 7.8) $0.5 \mathrm{mM}$ EDTA and $0.03 \%$ SDS (18). Cleavage activity was monitored continuously by detection of free 7-amido-4-methylcoumarin (AMC) using a fluorescence plate reader (Gemini; Molecular Devices, Sunnyvale, CA, USA) at $380 / 460 \mathrm{~nm}$ and $37^{\circ} \mathrm{C}$. As controls, the AMC $(2 \mu \mathrm{M})$ was incubated with the drugs in buffer I without the cell extracts, and measurements of proteasome function were corrected when necessary.

Quantitative polymerase chain reaction ( $q P C R)$. Total RNA was extracted from each experimental group using TRIzol ${ }^{\circledR}$ eagent (Invitrogen Life Technologies, Carlsbad, CA, USA), according to the manufacturer's instructions. RNA concentration was assessed spectrophotometrically at $260 \mathrm{~nm}$ (Thermo ND 2000; Thermo Scientific). Reverse transcription was performed on the isolated total RNA using a Reverse Transcription kit (Takara Bio, Inc., Otsu, Japan), and PCR was carried out using a Real Time PCR kit (Takara Bio, Inc.). Reverse transcription was performed at $65^{\circ} \mathrm{C}$ for $5 \mathrm{~min}, 30^{\circ} \mathrm{C}$ for $10 \mathrm{~min}, 42^{\circ} \mathrm{C}$ for $10-30 \mathrm{~min}$ and $92^{\circ} \mathrm{C}$ for $3 \mathrm{~min}$. PCR conditions were as follows: Denaturation at $94^{\circ} \mathrm{C}$ for $2 \mathrm{~min}$; amplification for 30 cycles at $94^{\circ} \mathrm{C}$ for $0.5 \mathrm{~min}$, annealing at $60^{\circ} \mathrm{C}$ for $0.5 \mathrm{~min}$, and extension at $72^{\circ} \mathrm{C}$ for $1 \mathrm{~min}$; followed by a terminal elongation step at $72^{\circ} \mathrm{C}$ for $10 \mathrm{~min}$, and was perfomed on a Bio-Rad CFX96 thermal cycler (Bio-Rad Laboratories, Inc.). U6 was amplified as an internal control and the $\mathrm{Ct}$ value of each PCR product was calculated, and the fold change was analyzed. The m-miR-423-5p, h-miR-423-5p, m-U6 and h-U6 primers were supplied by Riob Bio Technology (Guangzhou, China); the sequences were not supplied due to the rules of the company.

Detection of miR-423-5p downstream targets using western blot analysis. The cardiomyocytes were transfected with m-miR-423-5p or miR-Ctrl for 24 or $48 \mathrm{~h}$, as described above, following which the total protein was collected. The cells were lysed on ice for $30 \mathrm{~min}$ using radioimmunoprecipitation assay lysis buffer, containing $50 \mathrm{mM}$ Tris- $\mathrm{HCl}$ (pH 7.4), 1\% NP-40, $0.25 \%$ Na-deoxycholate, $150 \mathrm{mM} \mathrm{NaCl}, 1 \mathrm{mM}$ EDTA, $1 \mathrm{mM}$ phenylmethylsulfonyl fluoride, $1 \mu \mathrm{g} / \mathrm{ml}$ aprotinin, $1 \mu \mathrm{g} / \mathrm{ml}$ leupeptin, $1 \mu \mathrm{g} / \mathrm{ml}$ pepstatin, $1 \mathrm{mM} \mathrm{Na} \mathrm{VO}_{4}$ and $1 \mathrm{mM} \mathrm{NaF}$. The proteins $(20 \mu \mathrm{g})$ were separated by SDS-polyacrylamide gel electrophoresis (SDS-PAGE; Beyotime Biotechnology) and electronically transferred onto a polyvinylidene difluoride membrane (Millipore, Bedford, MA, USA). Following blocking with $5 \%$ skim milk powder in tris-buffered saline with Tween, the membranes were incubated with recommended dilution primary antibodies against OGT (rabbit polyclonal; 1:800 for $1 \mathrm{~h}$ at $37^{\circ} \mathrm{C}$; cat. no. sc-32921; Santa Cruz Biotechnology, Inc. Dallas, TX, USA), phosphorylated (p)-adenosine monophosphate (AMP)-activated protein kinase (AMPK; cat. no. 4188; 1:1,000), AMPK (cat. no. 2532; 1:1,000) p53 (cat. no. 2527; $1: 1,200$ ) and caspase 3 (cat. no. 9662; 1:600) for $1 \mathrm{~h}$ at $37^{\circ} \mathrm{C}$ (all purchased from Cell Signaling Technology, Inc., Danvers, MA, USA), and GAPDH (cat. no. sc-25778; 1:5,000; Santa Cruz Biotechnology, Inc.). This was followed by incubation with peroxidase-conjugated secondary antibodies (Abcam,
A

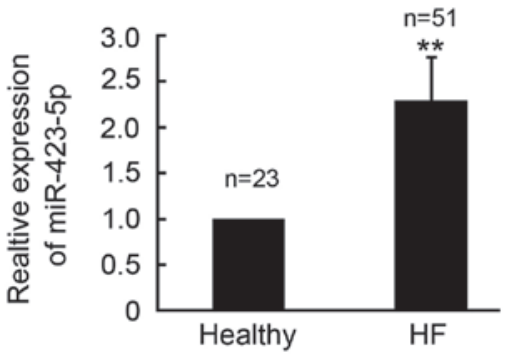

B

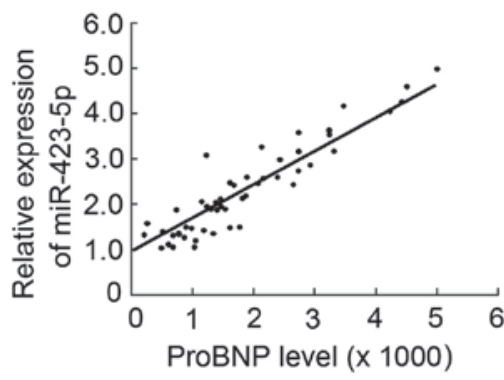

Figure 1. Expression of miR-423-5p in plasma from patients with CHF. (A) Plasma from 51 patients with $\mathrm{CHF}$ and 23 healthy controls was collected and the expression of miR-423-5p was determined using quantitative polymerase chain reaction analysis. Data are presented as the mean \pm standard error of the mean. ${ }^{* *} \mathrm{P}<0.01$, vs. healthy controls. (B) Spearman's correlation between miR-423-5p and proBNP (P<0.01). miR-423-5p, microRNA-423-5p; CHF, congestive heart failure; proBNP, pro-brain natriuretic peptide.

Cambridge, MA, USA). The peroxidase-labeled bands were visualized using an enhanced chemiluminescence kit (Pierce Biotechnology, Inc.). The ratio of OGT, p-AMPK, AMPK, p53 and caspase 3/GAPDH were calculated using densitometry.

Terminal deoxynucleotidyl transferase-deoxyuridine triphosphate nick-end labeling (TUNEL) assay. In order to detect apoptotic cells in cardiomyocytes following transfection with miR-423-5p and miR-Ctrl, a TUNEL assay was performed using a DeadEndTM Fluorometric TUNEL system (Promega Corporation), according to the manufacturer's instructions. Cell nuclei with dark green fluorescent staining were defined as TUNEL-positive nuclei, which were visualized using a fluorescence microscope (DTX500; Nikon Corporation, Tokyo, Japan). In order to quantify the TUNEL-positive cells, the number of green fluorescence-positive cells were counted in randomly selected fields at x200 magnification. The cell nuclei were then counter-stained with 4',6-diamidino-2-phenylindole (Beyotime Biotechnology).

Statistical analysis. Statistical comparisons of all the results were analyzed using a one-way analysis of variance. Statistical analyses were performed using SPSS version 19.0 (IBM SPSS, Armonk, NY, USA). Values are expressed as the mean \pm standard error of the mean. $\mathrm{P}<0.05$ was considered to indicate a statistically significant difference.

\section{Results}

miR-423-5p is upregulated in patients with CHF. In order to investigate whether the expression of miR-423-5p was altered in patients with $\mathrm{CHF}$, plasma samples from patients and healthy controls were collected and qPCR was performed to determine 
A

5'-UUUCAGCCCUUGACUGCCCCUCC-3' OGT 3' UTR
||||||||
3'-UUUCAGAGCGAGAGACGGGGAGU-5' m-miR-423-5p
$\mid$ | || || | |
5'-UUUCAGCCCUUGACUGACCCGCC-3' OGT 3' UTR mutant

B

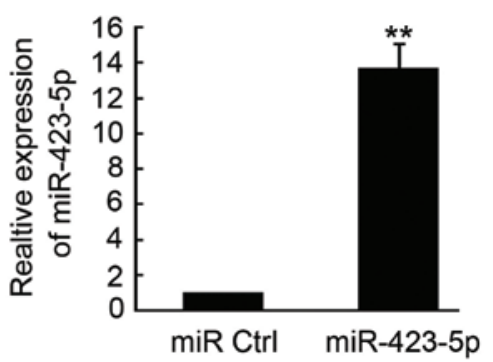

C

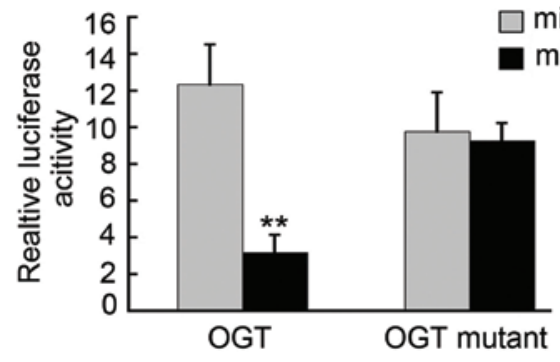

Figure 2. m-OGT is a target of m-miR-423-5p. (A) Bioinformatic analysis revealed that m-OGT 3'-UTR contained one predicted m-miR-423-5p binding site. The predicted duplex formations between m-OGT 3'UTR and m-miR-423-5p are depicted. Blue indicates sites of target mutagenesis. (B) Quantitative polymerase chain reaction analysis of m-miR-423-5p in the cardiomyocytes following transfection with m-miR-423-5ps. (C) Relative repression of luciferase activity following transfection of the OGT and OGT mutant constructs into the miR-423-5p- and miR-Ctrl-transfected cells. Data are presented as the mean \pm standard error of the mean. ${ }^{* *} \mathrm{P}<0.01$, vs. miR-Ctrl. m-miR-423-5p, mouse microRNA-423-5p; miR-Ctrl, control plasmid; OGT, O-GlcNAc transferase; UTR, untranslated region.

A

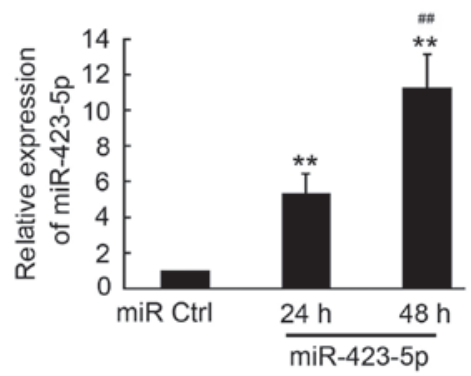

B

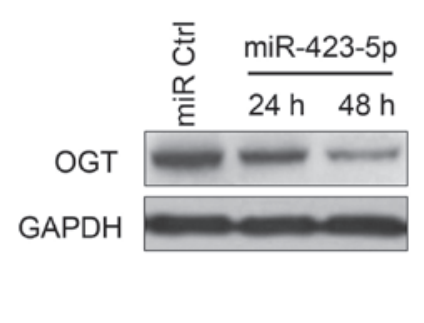

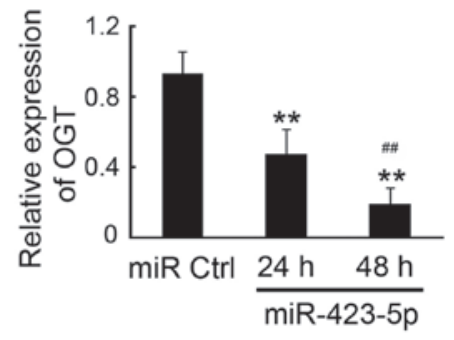

D

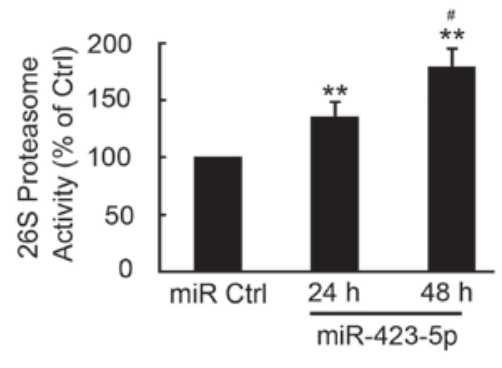

Figure 3. m-miR-423-5p regulates m-OGT and the expression of downstream targets of OGT. (A) m-miR-423-5p was transfected into the cardiomyocytes and quantitative polymerase chain reaction was used to analyze its expression 24 and $48 \mathrm{~h}$ post-transfection. Western blotting and densitometric analysis were used to determine the (B) protein expression of OGT and of (C) AMPK and p-AMPK in cardiomyocytes transfected with miR-423-5p for 24 and 48 h. GAPDH was used as a loading control. (D) Total protein from the miR-423-5p-transfected cardiomyocytes was collected at 24 and $48 \mathrm{~h}$ post-transfection and used to examine $26 \mathrm{~S}$ proteasome activity. Data are presented as the mean \pm standard error of the mean. ${ }^{* *} \mathrm{P}<0.01$, vs. miR-Ctrl, ${ }^{\#} \mathrm{P}<0.05$ and ${ }^{\# \#} \mathrm{P}<0.01$, vs. miR-423-5p transfected for $24 \mathrm{~h}$. $\mathrm{m}-\mathrm{miR}-423-5 \mathrm{p}$, mouse microRNA-423-5p; miR-Ctrl, control plasmid; OGT, O-GlcNAc transferase; AMPK, adenosine monophosphate-activated protein kinase; p-, phosphorylated.

the expression levels of circulating miR-423-5p in the patients with $\mathrm{CHF}$ and the healthy controls. The results demonstrated that the expression of miR-423-5p was upregulated in the patients, with CHF compared with the healthy controls (Fig. 1A). The circulating protein levels of the proBNP biomarker of CHF were also determined and the results indicated that the circulating levels of miR-423-5p were positively correlated with the expression of proBNP and the CHF classification (Fig. 1B).
OGT is a target of $m-m i R-423-5 p$. To determine the targets of $\mathrm{m}-\mathrm{miR}-423-5 \mathrm{p}$, a number of potential target proteins in a database library were screened to identify potential miRNA binding seed sequences within the 3'-UTR. Using bioinformatic analyses (Targetscan, microRNA.org and microRNASeq), it was found that m-OGT was a potential candidate target for m-miR-423-5p (Fig. 2A). In order to verify this, m-OGT-3'UTR containing an miR-423-5p binding site were cloned downstream 
A

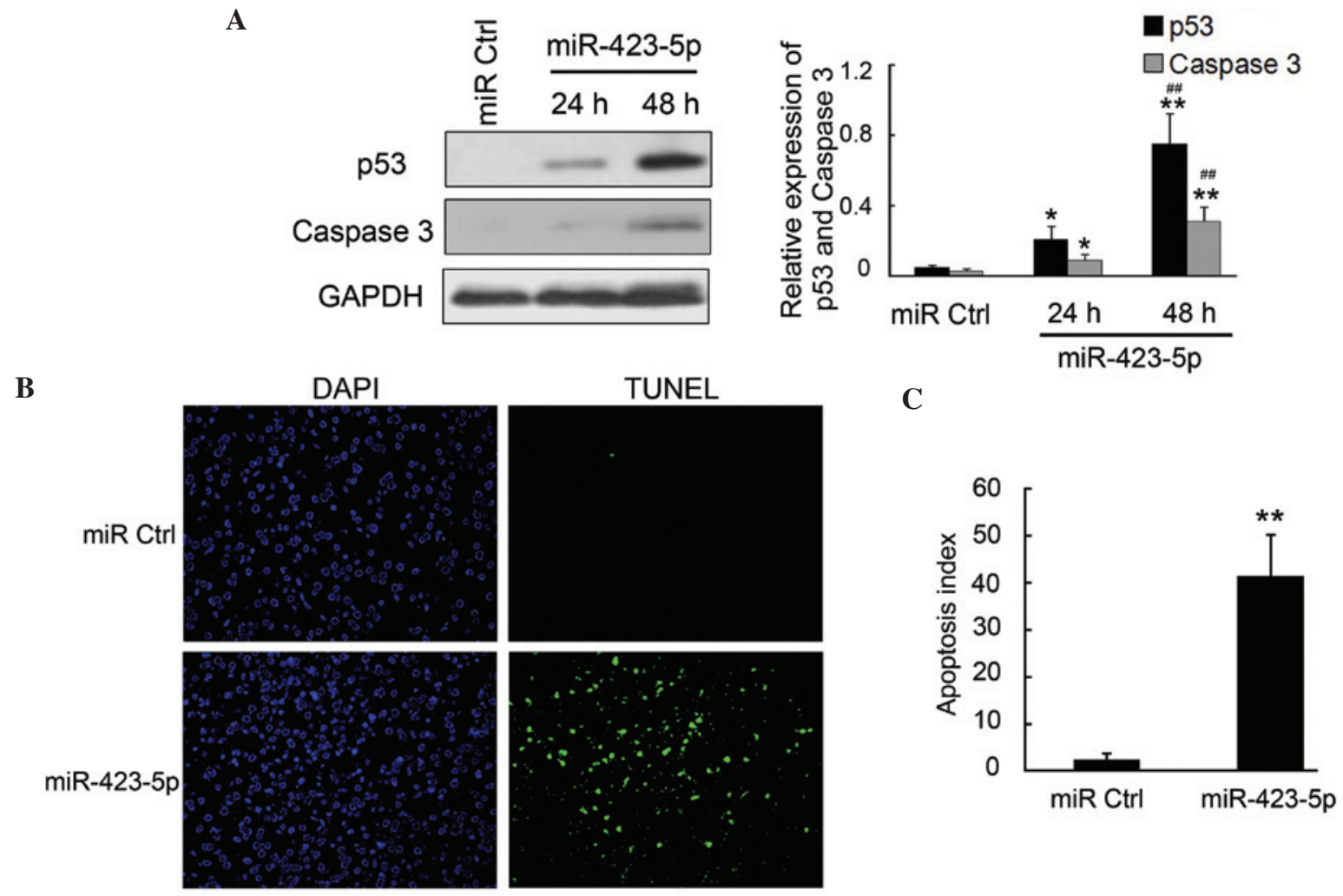

Figure 4. miR-423-5p induces apoptosis in cardiomyocytes. (A) miR-423-5p was transfected into cardiomyocytes and total protein was collected 24 and $48 \mathrm{~h}$ post-transfection. Western blotting and densitometric analyses were used to determine the protein expression levels of p53 and caspase-3. GAPDH was used as a loading control. (B) Cardiomyocyte apoptosis was determined using a TUNEL assay following transfection with miR-423-5p for 48 h. Positive cells are indicated in green and DAPI staining indicates the whole cells (magnification, x200). (C) Quantitative analysis of apoptosis (apoptotic index = number of apoptotic cells/total number of cells). Data are presented as the mean \pm standard error of the mean. ${ }^{*} * \mathrm{P}<0.01$, vs. miR-Ctrl, ${ }^{\#} \mathrm{P}<0.05$ and ${ }^{\# \#} \mathrm{P}<0.01$, vs. miR-423-5p transfected for $24 \mathrm{~h}$. miR-423-5p, microRNA-423-5p; miR-Ctrl, control plasmid; TUNEL, terminal deoxynucleotidyl transferase-deoxyuridine triphosphate nick-end labeling; DAPI, 4',6-diamidino-2-phenylindole.

of the luciferase open reading frame. The m-OGT-3'UTR mutant, which contained a mutated m-miR-423-5p binding site, was also introduced into the luciferase construct. The plasmid expressing m-miR-423-5p was transfected into the cardiomyoctes, and puromycin (Beyotime Biotechnology) was used to select the stable expression cells. qPCR analysis confirmed that m-miR-423-5p was upregulated in these cells (Fig. 2B). The luciferase-OGT-3'UTR and luciferase-OGT-3'UTR mutant constructs were then transfected into the 293 cells stably expressing m-miR-423-5p. Following 4-6 h transfection, the expression of luciferase in the OGT-3'UTR constructs was significantly downregulated compared with that of the miR-Ctrl, whereas luciferase activity was unaffected in the cells transfected with the mutant construct (Fig. 2C).

m-miR-423-5p regulates the expression levels of OGT and downstream targets of OGT in cardiomyocytes. The effects of m-miR-423-5p transfection were investigated in cardiomyocytes from mice due to the limited use of human cardiomyocytes in investigations. The plasmid encoding $\mathrm{m}-\mathrm{miR}-423-5 \mathrm{p}$ was transfected into the mouse cardiomyocytes and incubated for 24 or $48 \mathrm{~h}$. The cells were then collected and qPCR was performed to determine the expression of levels of $\mathrm{m}-\mathrm{miR}-423-5 \mathrm{p}$. $\mathrm{m}-\mathrm{miR}-423-5 \mathrm{p}$ was significantly upregulated at 24 and $48 \mathrm{~h}$ post-transfection compared with the miR-Ctrl (Fig. 3A). There was significantly increased expressed of miR-423-5p at $48 \mathrm{~h}$ compared with that at $24 \mathrm{~h}(\mathrm{P}<0.01)$. Western blotting and densitometric analysis indicated that the OGT in the cardiomyocytes was significantly inhibited by m-miR-423-5p (Fig. 3B). The activities of AMPK and the $26 \mathrm{~S}$ proteasome, as the downstream targets of OGT, were also determined. The results demonstrated that the phosphorylation of AMPK was inhibited by $\mathrm{m}-\mathrm{miR}-423-5 \mathrm{p}$, which was comparable with the reduction in OGT (Fig. 3C). However, no significant change was observed in the expression of total AMPK. Furthermore, the activities of the $26 \mathrm{~S}$ proteasome increased significantly by $1.35 \pm 0.12-$ and $1.79 \pm 0.16$-fold 24 and 48 h post-transfection with m-miR-423-5p, respectively, compared with miR-Ctrl.

m-mir-423-5p induces apoptosis in cardiomyocytes through upregulation of p53 and caspase-3. Cardiomyocyte apoptosis has been previously reported as the primary cause of CHF (19); therefore, the present study examined the apoptosis and the expression of apoptosis-associated proteins using TUNEL and western blot analysis. The results of the western blot analysis revealed that the pro-apoptotic proteins $\mathrm{p} 53$ and caspase-3, which are the downstream targets of AMPK, were significantly upregulated by m-miR-423-5p in the cardiomyocytes (Fig. 4A and B). The TUNEL assays indicated that the apoptotic rate was significantly increased in the m-miR-423-5p-transfected cardiomyocytes, compared with the miR-Ctrl group (Fig. 4C and D). Overall, these results demonstrated that the increased expression of m-miR-423-5p in cardiomyocytes significantly induced cell apoptosis via the upregulation of p53 and caspase-3. 


\section{Discussion}

The results of the present study demonstrated that the expression of miR-423-5p was positively correlated with CHF diagnosis and the plasma levels of proBNP. In addition, the results indicated that OGT was a target of miR-423-5p; therefore, it was hypothesized that miR-423-5p directly targeted the OGT 3'-UTR, inhibiting the expression of OGT and inducing apoptosis in the cardiomyocytes. Furthermore, the inhibition of OGT by miR-423-5p significantly decreased the expression of p-AMPK and upregulated the activity of the $26 \mathrm{~S}$ proteasome and the protein expression levels of pro-apoptotic p53 and caspase-3. The TUNEL analysis also revealed increased apoptosis in the cardiomyocytes following transfection with miR-423-5p.

Previous evidence suggests that circulating miRNAs may be used as stable blood-based biomarkers in cancer (12). In particular, the expression levels of miR-1 and miR-208 cardiac miRNAs are elevated in plasma following myocardial injury, which has been suggested to occur due to the release of these miRNAs from the damaged cardiac cells $(20,21)$. The results of a previous array study reported that miR-423-5p is upregulated in the failing human myocardium (13) and circulating levels of miR-423-5p have been associated with proBNP and ejection fractions in this patient groups (14). However, the use of miR-423-5p as a biomarker for systemic ventricular and left ventricular remodeling has been unsuccessful $(15,16)$. The results of the present study demonstrated that circulating levels of miR-423-5p were upregulated in theCHF patients and were associated with the experssion of proBNP. In addition, the expression levels of miR-423-5p in the plasma may also provide novel biomarker for CHF. However, further clinical investigation is required in order to confirm this hypothesis.

The post-translational $\beta$-O-linkage of $\mathrm{N}$-acetylglucosamine (O-GlcNAc) to cellular proteins is an important signaling pathway, which has been implicated in the pathophysiology of cardiovascular disease (22). OGT enables the addition of $\mathrm{O}-\mathrm{GlcNAc}$ to proteins and is required for cell division and embryogenesis (23). Cardiomyocyte ablation of OGT induces heart failure, and OGT is required for the endogenous compensatory response to infarct-induced heart failure (24). AMPK is present in all mammalian cells and is a sensor of cellular energy (25) and cellular redox status (26). As a downstream target of OGT, the reduction of p-AMPK by OGT in endothelial cells upregulates proteasome activity (27). The results of the present study indicated that OGT was a target of miR-423-5p; and that the expression of miR-423-5p in mouse cardiomyocytes significantly inhibited the expression of OGT and phosphorylation of the downstream target, AMPK. Of note, 26S proteasome activity and the expression levels of the p53 and caspase-3 pro-apoptotic proteins were markedly increased. Furthermore, according to the molecular changes observed in the TUNEL assay, the apoptotic rate of cells was increased in cardiomyocytes transfected with miR-423-5p.

\section{References}

1. Dassanayaka S and Jones SP: O-GlcNAc and the cardiovascular system. Pharmacol Ther 142: 62-71, 2013.

2. Shafi R, Iyer SP, Ellies LG, O'Donnell N, Marek KW, Chui D, et al. The O-GlcNAc transferase gene resides on the $\mathrm{X}$ chromosome and is essential for embryonic stem cell viability and mouse ontogeny. Proc Natl Acad Sci USA 97: 5735-5739, 2000.
3. Watson LJ, Facundo HT, Ngoh GA, Ameen M, Brainard RE, Lemma KM, et al: O-linked beta-N-acetylglucosamine transferase is indispensable in the failing heart. Proc Natl Acad Sci USA 107: 17797-17802, 2010 .

4. Mann DL and Bristow MR: Mechanisms and models in heart failure: the biomechanical model and beyond. Circulation 111: 2837-2849, 2005.

5. Seidman JG and Seidman C: The genetic basis for cardiomyopathy: from mutation identification to mechanistic paradigms. Cell 104: $557-567,2001$.

6. Januzzi JL, van Kimmenade R, Lainchbury J, Bayes-Genis A, Ordonez-Llanos J, Santalo-Bel M, et al: NT-proBNP testing for diagnosis and short-term prognosis in acute destabilized heart failure: an international pooled analysis of 1256 patients: the International Collaborative of NT-proBNP Study. Eur Heart J 27: 330-337, 2006.

7. van Kimmenade RR, Pinto YM and Januzzi JL Jr: Importance and interpretation of intermediate (gray zone) amino-terminal pro-B-type natriuretic peptide concentrations. Am J Cardiol 101: 39-42, 2008.

8. Ambros V: The functions of animal microRNAs. Nature 431: 350-355, 2004.

9. van Rooij E, Marshall WS and Olson EN: Toward microRNA-based therapeutics for heart disease: the sense in antisense. Circ Res 103: 919-928, 2008.

10. Chen X, Ba Y, Ma L, Cai X, Yin Y, Wang K, et al: Characterization of microRNAs in serum: a novel class of biomarkers for diagnosis of cancer and other diseases. Cell Res 18: 997-1006, 2008.

11. Lawrie CH, Gal S, Dunlop HM, Pushkaran B, Liggins AP, Pulford K, et al: Detection of elevated levels of tumour-associated microRNAs in serum of patients with diffuse large B-cell lymphoma. Br J Haematol 141: 672-675, 2008.

12. Mitchell PS, Parkin RK, Kroh EM, Fritz BR, Wyman SK, Pogosova-Agadjanyan EL, et al: Circulating microRNAs as stable blood-based markers for cancer detection. Proc Natl Acad Sci USA 105: 10513-10518, 2008.

13. Thum T, Galuppo P, Wolf C, Fiedler J, Kneitz S, van Laake LW, et al: MicroRNAs in the human heart: a clue to fetal gene reprogramming in heart failure. Circulation 116: 258-267, 2007.

14. Tijsen AJ, Creemers EE, Moerland PD, de Windt LJ, van der Wal AC, Kok WE, et al: MiR423-5p as a circulating biomarker for heart failure. Circ Res 106: 1035-1039, 2010.

15. Bauters C, Kumarswamy R, Holzmann A, Bretthauer J, Anker SD, Pinet F, et al: Circulating miR-133a and miR-423-5p fail as biomarkers for left ventricular remodeling after myocardial infarction. Int J Cardiol 168: 1837-1840, 2013.

16. Tutarel O, Dangwal S, Bretthauer J, Westhoff-Bleck M, Roentgen P, Anker SD, et al: Circulating miR-423_5p fails as a biomarker for systemic ventricular function in adults after atrial repair for transposition of the great arteries. Int J Cardiol 167: 63-66, 2013.

17. Fekete MR, McBride WH and Pajonk F: Anthracyclines, proteasome activity and multi-drug-resistance. BMC Cancer 5: 114, 2005.

18. Rosamond WD, Chang PP, Baggett C, et al: Classification of heart failure in the atherosclerosis risk in communities (ARIC) study: A comparsion of diagnostic criteria. Circ Heart Fail 5: 152-159, 2012.

19. Yang C, Liu Z, Liu K and Yang P: Mechanisms of Ghrelin anti-heart failure: Inhibition of Ang II-induced cardiomyocyte apoptosis by down-regulating AT1R expression. PLoS One 9: e85785, 2014.

20. Ai J,Zhang R, Li Y,Pu J, Lu Y, Jiao J, et al: Circulating microRNA-1 as a potential novel biomarker for acute myocardial infarction. Biochem Biophys Res Commun 391: 73-77, 2010.

21. Ji X, Takahashi R, Hiura Y, Hirokawa G, Fukushima Y and Iwai N: Plasma miR-208 as a biomarker of myocardial injury. Clin Chem 55: 1944-1949, 2009.

22. Dassanayaka S and Jones SP: O-GlcNAc and the cardiovascular system. Pharmacol Ther 142: 62-71, 2014.

23. Shafi R, Iyer SP, Ellies LG, O'Donnell N, Marek KW, Chui D, et al: The O-GlcNAc transferase gene resides on the $\mathrm{X}$ chromosome and is essential for embryonic stem cell viability and mouse ontogeny. Proc Natl Acad Sci USA 97: 5735-5739, 2000.

24. Watson LJ, Facundo HT, Ngoh GA, Ameen M, Brainard RE, Lemma KM, et al: O-linked beta-N-acetylglucosamine transferase is indispensable in the failing heart. Proc Natl Acad Sci USA 107: 17797-17802, 2010.

25. Hardie DG: AMPK and Raptor: matching cell growth to energy supply. Mol Cell 30: 263-265, 2008.

26. Zou MH and Wu Y: AMP-activated protein kinase activation as a strategy for protecting vascular endothelial function. Clin Exp Pharmacol Physiol 35: 535-545, 2008.

27. Xu J, Wang S, Viollet B and Zou MH: Regulation of the proteasome by AMPK in endothelial cells: the role of O-GlcNAc transferase (OGT). PloS one 7: e36717, 2012. 